\title{
Starry, starry night
}

\section{The Heavens on Fire: The Great Leonid Meteor Storms \\ by Mark Littmann \\ Cambridge University Press: 1998. 348 pp. $\mathfrak{£} 25, \$ 39.95$ \\ Owen Gingerich}

Early on the morning of 13 November 1833 , residents of eastern North America who happened to be awake (or awakened!) were treated to an astonishing, unexpected and fearsome sight. The sky was filled with shooting stars more numerous than one could count, a veritable storm of meteors.

Historical researches in the days that followed revealed that the event was not as singular as had first been supposed. A similar display had been seen from Venezuela by Alexander von Humboldt 34 years earlier, on 12 November 1799. And another interesting fact emerged: based on a bright meteor observed from two locations, the Yale mathematics professor Denison Olmsted calculated that it had burned out 30 miles above the Earth.

The meteorologists were offended. Meteors were their turf, and they thought they knew that the atmosphere did not extend that high. The notion that the meteors might be periodic smacked of astronomy. How absurd!

By 1866 meteors were well established as cosmic phenomena, and astronomers keenly awaited another meteor storm. This time it was Europe's turn for a dazzling display, though by all accounts not one to match the 1833 spectacle. And before the year was out, the Italian astronomer Giovanni Schiaparelli matched the orbit of another meteor shower, the annual August shooting stars that seemed to radiate from the constellation Perseus, with that of the Comet 1862 III. He could not, however, identify the November meteors (by this time called the Leonids) with any known comet. The reason was that the comet, Tempel-Tuttle, 1866 I, had only just been found, and its orbit was not immediately known.

Needless to say, astronomers eagerly awaited a repeat performance in 1899 . The press played the story with a flourish, and turned upon the scientists with scorn when the great show did not materialize. Jupiter, the real villain, had perturbed the comet and its stream of dusty debris. No substantial performance arrived with 1933, and then surprise! — a grand storm in 1966 for those in the western United States stubborn enough to have stayed up into the wee hours of the morning of 17 November.

Now, to have made a book of all this requires a fertile and informed imagination, and this Mark Littmann, professor of science journalism at the University of Tennessee, has brought to his project in abundance.

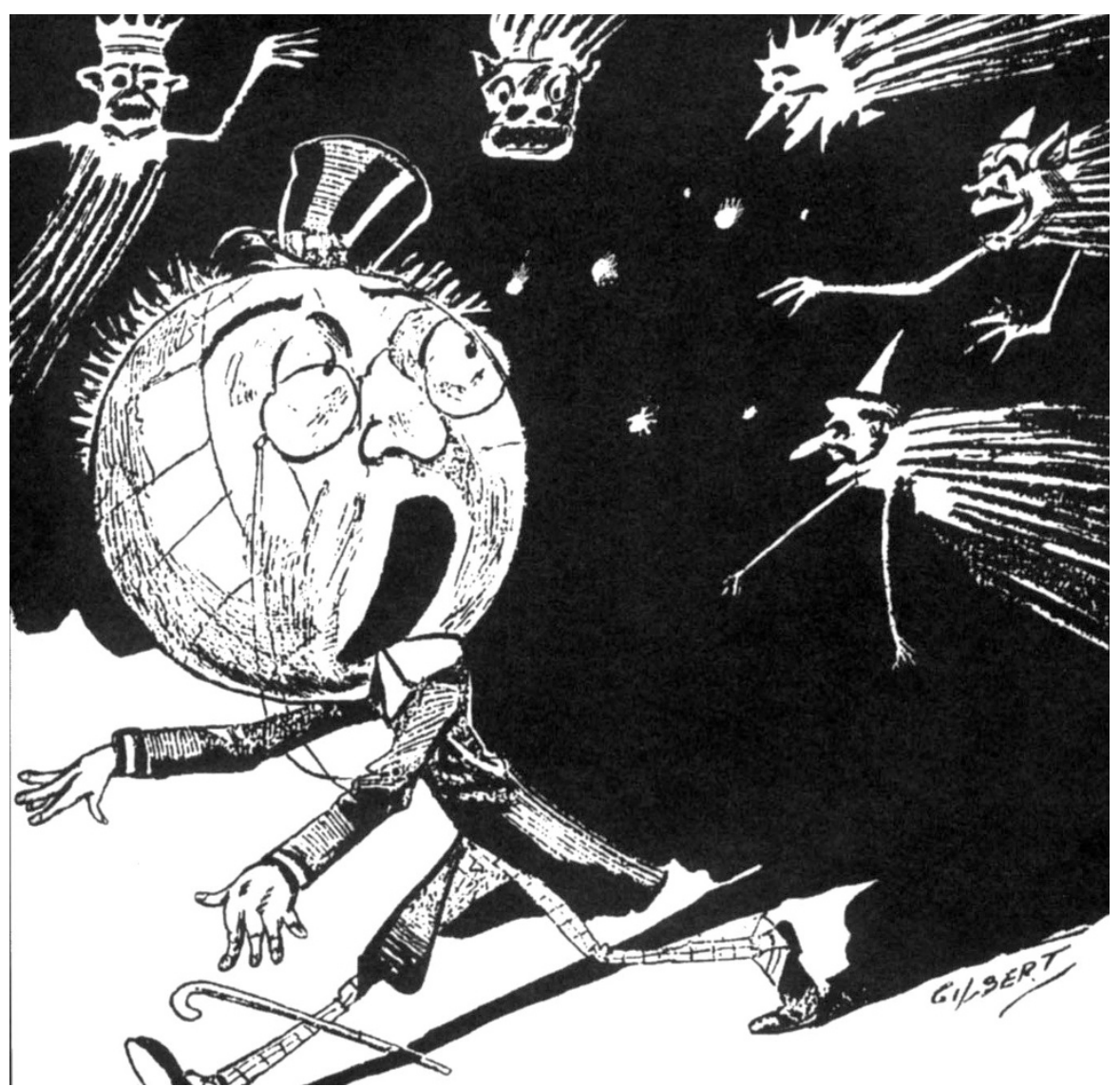

High expectations: the Denver Post reports the failed appearance of the predicted 1899 meteor storm.

Littmann tells his story with real flair, and expansively enough to teach a great deal of meteor, comet and meteorite astronomy in the process. There is considerable technical detail, mostly in side-bars, so the book is altogether satisfying both as a quick, informative read and also as a reference source. In addition, it is filled with well-chosen illustrations.

For those who would like to observe some meteors, and even to photograph them, the instructions are here in Littmann's book. Showers come every year, but storms rarely. Still, the Leonids are not the only sequence going. I vividly remember catching the Giacobini-Zinner storm in 1946, counting 1,500 meteors in three hours despite bright moonlight — this is a conveniently placed shower that comes in the evening rather than in the morning skies (and Littmann explains why).

Two aspects of the book require special comment. First, in treating the historical material, Littmann frequently adds in italics our contemporary view of the matter. Professional historians of science tend to frown on this as whiggishness, but I found it entirely appropriate for a general audience. Second, the book is overfilled with grey side-bars enriching the historical or technical material. The book's designer may have thought they made quite beautiful patterns, but as a reader
I found them maddeningly distracting, especially when they broke up the narrative flow right in the middle of sentences.

Another anniversary of Comet TempelTuttle is now upon us. After being lost for a century, and presumed to have disintegrated into the orbital debris responsible for the meteor shower, the comet was rediscovered in 1966. It again faded into invisibility on its long trip out past Uranus, but on this round it was caught nearly a year before its perihelion passage (closest approach to the Sun) by astronomers using the 10-metre Keck telescope atop Mauna Kea in Hawaii. Last November, some observers viewed a satisfying display, though hardly a meteor storm.

Perhaps 1999 will furnish a more memorable show. If so, it will be the last good opportunity in a century, because the placement of the stream will not be favourable again until 2098, and after that 2131 and 2164 are not as promising. "Those are the Leonids' last gasp," Littmann writes, for the orientation of the orbit will slowly drift away from the Earth. There were no great Leonid storms before the ninth century, and there will be no more beyond 2164 .

Owen Gingerich is at the Harvard-Smithsonian

Center for Astrophysics, Cambridge, Massachusetts 02138, USA. 